\title{
Analysis of Control and Multimedia Real-Time Traffic over SIP and RTP on 802.11n Wireless Links for Utilities Networks
}

\author{
Salvador Santonja-Climent, David Todoli-Ferrandis, \\ Teresa Albero-Albero, Victor-M. Sempere-Payá, \\ Javier Silvestre-Blanes \\ ITI. Universitat Politècnica de Valencia. \\ $\{$ ss,dt,talbero,vsempere,jsilves\}@iti.upv.es
}

\author{
Jesus Alcober \\ Telematics Engineering Department \\ Universitat Politecnica de Catalunya. \\ jesus.alcober@upc.edu
}

\begin{abstract}
Urban facility interconnection networks require a robustness and reliability usually found in public networks or private cable and radio networks in licensed bands. Both factors mean an increase in costs, which also increase as the size of the network increases. The recent rise in use of wireless technologies in open wavebands has attracted the interest of the industry in the spread of these types of links, even though they have a lower level of robustness and reliability, which must be improved using communication mechanisms and protocols. This aspect directly affects real time applications, such as VoIP and video-streaming, and determines the viability of these systems in real installations. This paper evaluates the performance of control and real time multimedia traffic in the $5 \mathrm{GHz}$ band through the use of the SIP (Session Initiation Protocol) and RTP (Real-Time Transport Protocol) protocols, in a laboratory testbed.
\end{abstract}

\section{Introduction}

Waste water networks in metropolitan areas operate over a network of distributed stations which fulfill a double function of monitoring and actions, sending environmental and water quality data to the central station, and receiving orders to be executed for sluices and pumps. The traffic generated by these stations can be grouped into: control and telemetry traffic, voice traffic (between operators and central) and video traffic (coming from cameras that monitor water levels, obstructions or collectors status). In general, the communication from the central to the remote stations is carried out using heterogeneous networks, which combine the coverage and performance of public networks (both wired and wireless) and private networks. The use of private networks is interesting in those segments where they have spread enough to be technically and economically viable, which will mean a considerable saving in costs of use and greater control of the network. Industrial networks giving support to these types of ins- tallations are characterized by high reliability, adaptability and scalability. However, the use of fieldbuses and other wired technology are limited to local use, which has sparked interest in the use of communication technology with wider coverage for interconnection in urban areas. It is usual in this context to see the use of wireless technology over licensed bands, which allows the use of an interference-free channel as well as high transmission power, which in turn means greater range and a higher level of signal/noise relationship in the receivers, giving greater reliability. However, the current trend is to use open bands, such as $868 \mathrm{MHz}, 2.4 \mathrm{GHz}$ or $5 \mathrm{GHz}$. The use of these bands offers significant benefits in terms of installation and using costs, as there is an extremely active market which is generating a huge quantity of new technology and products. However, there are certain disadvantages which must be considered, such as the reduction in transmission power limits, which translates into a lower range and weaker signal/noise relationship. Another disadvantage is that the band is shared with other users so on occasions may be saturated, as $2.4 \mathrm{GHz}$ band. Industrial networks require high reliability and availability in communications, which means it is necessary to study mechanisms which can provide better guarantees for the traffic in these types of technologies. In this paper, the behavior of this three types of traffic necessary for the interconnection of remote stations of a real metropolitan waste water system using 802.11n [2] radio technology and the SIP [4] and RTP [5] session and transport protocols, is studied and evaluated. The experiments were carried out on a laboratory testbed, in order to obtain greater control over the tests, and further experiments will be conducted in a real exterior facility.

The need for new services in Valencia's waste water system, such as voice and video, has led to a gradual increase of means of access to public networks, installed by tele-operators to give service to the stations, which involves a monthly cost as well as the externalization of control of the network. With the objective of using a private network and reducing costs, we have used a laboratory testbed to recreate 1 and 2 hop links with 
802.11n, and carried out an initial evaluation of the services offered over this real network. Fig. 1 shows an aerial photograph of the Central Control Station (CCS) and the nearby installations with which it must maintain fixed links. In this image, we can see 1 hop links (stations 1 and 2) and 2 hop links (stations 3, 4 or 5). The use of the $5 \mathrm{GHz}$ band means there are no costs involved, and there is less saturation than with the $2.4 \mathrm{GHz}$ band, as well as a greater quantity of available channels, making WiMax and 802.11n good candidates for these applications [6].

\section{Scenario}

The testbed is made up of 3 Linksys WRT602N routers, with channels of $40 \mathrm{MHz}$ bandwidth and a physical distance of 5 meters between each. Using virtual card configuration and bridge mode, they were connected by 2 hops at the data link layer level, obtaining a high capacity trunk with throughputs of up to $80 \mathrm{Mbps}$ from end to end. In order to create a situation as close to real as possible, the SIP server and a client are connected to one end of the trunk, corresponding to the central control station, and the second client is located in the intermediate router or at the other end of the trunk, in order to carry out tests of 1 and 2 hops via remote station (Fig. 2). The server uses OpenSIPS 1.6.2 and Asterisk 1.2.21.2 to offer these services, and clients use free softphone X-Lite 2.0 for Linux environments and 4.0 for Windows.

\section{Technologies used}

The $802.11 \mathrm{n}$ standard was ratified by the IEEE organization on $09 / 11 / 2009$, with a speed of up to $600 \mathrm{Mbps}$ in the physical layer. Its range and quality are improving thanks to new MIMO (Multiple Input Output) technology, which allows it to use several channels simultaneously to send and receive data, thanks to the incorporation of several antennas. The use of OFDM modulation offers greater resistance to interference in the channel. $802.11 \mathrm{n}$ can work in two frequency bands: $2.4 \mathrm{GHz}$ and $5 \mathrm{GHz}$, and at 20 or $40 \mathrm{MHz}$. Thanks to techniques to improve interference resistance, transmission speed and bandwidth, this new standard is becoming a real alternative, both economically and in terms of performance, to WiMaX for links of medium distance between urban installations.

Session Initiation Protocol, developed by the working group MMUSIC (Multiparty MUltimedia SessIon Con-

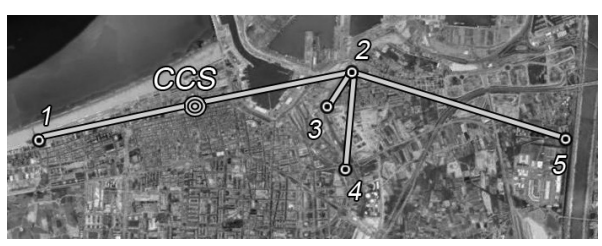

Figure 1. Purification Water Facilities trol) of the IETF (Internet Engineering Task Force), is a signaling protocol for interactive multimedia sessions. SIP is a protocol oriented toward the internet, and which can deal with mobile users. It has some extremely interesting characteristics, such as low processing needs, authentication and encryption systems, modularity, extendibility and simplicity of code and network scalability. SIP is the protocol that deals with establishing and finishing the communication, as well as negotiating the necessary parameters for this communication, such as the compression algorithms used, sampling frequencies and multimedia characteristics, pricing and information security. SDP (Session Description Protocol) is used to describe the content of the session, and RTP as the carrier for the voice and video of the session. The choice of SIP + RTP over UDP for the transfer of multimedia content offers a powerful call routing and control system between clients, and ensures a constant flow and organized delivery thanks to temporal marking techniques for the synchronization of the connection and reorganization of packets received using RTCP (RTP Control Protocol).

\section{Evaluation}

\subsection{Control Traffic}

Control traffic is made up of short sequences which carry readings from sensors or initiate actions over the PLCs (Programmable Logic Controller) of the station. In our case the station's largest frame would be 592 bytes of payload, this can be transmitted in a single Ethernet frame. Evaluation must consider the delay between transmission and reception (latency) and the percentage of losses. This can be done using the pingSIP tool [1] which sends a SIP message of type OPTION which circulates at the session level between origin and destination and diagnoses the proper functioning of the end to end link and across all levels involved. In contrast to ping, pingSIP does not suffer from blocks in routers with NAT and reaches directly the destination device and requires greater processing in the level of layer in which it is operating. Fig. 3 shows the results from 300 pingSIP tests for one and two hops, where a loss percentage of $0 \%$ was obtained in both cases. For one hop, $77 \%$ of the values are concentrated at around $2.88 \mathrm{~ms}$, with an arithmetic average of $5.79 \mathrm{~ms}$ (marked in the figure with a dotted line) and a typical deviation of $5.73 \mathrm{~ms}$. For two hops, the latency was concentrated at around $3.05 \mathrm{~ms}$, the arithmetic average was $6.52 \mathrm{~ms}$ and the typical deviation was $7.49 \mathrm{~ms}$. These latency values, in the order of a few milliseconds, are negligible for the tar-

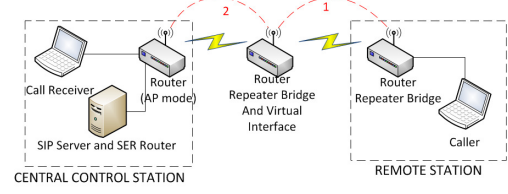

Figure 2. Testbed 


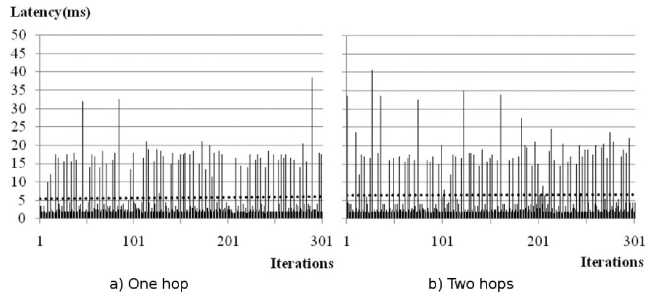

Figure 3. PingSIP results

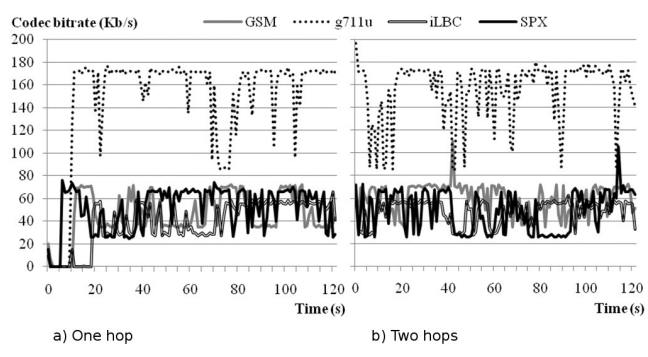

Figure 4. Audio Codecs throughput

get control applications, which demand latencies of several seconds, and so the system fulfilled all expectations.

\subsection{Voice Traffic}

Voice traffic needs certain parameters in order for the conversation to be fluent. The recommended maximum thresholds are latencies of $150 \mathrm{~ms}$ (according to ITU recommendation G.114), $100 \mathrm{~ms}$ of jitter and 1\% losses. The codec used is critical in this type of application as, due to the digitalization and compression of voice used [3], the frequency of transmission of frames as well as their size varies. The codec itself therefore generates a fixed delay in frame transmission due to the need to fill it with part of the conversation. The codecs evaluated and their characteristics are shown in Table 1. Figs. 4 show that codec G.711u uses more bandwidth, due to a higher bitrate for a better quality, with less compression complexity. However, the other codecs use less bandwidth and tolerate networks with less capacity. GSM is a codec widely used in mobile telephones, whose voice quality is low compared to more modern codecs. In iLBC (internet Low Bitrate Codec), the loss or delay of packets is balanced out through degradation of voice quality. iLBC is a good codec for environments with high losses, but with lower performance in controlled environments or those with low losses. SPEEX maintains a variable codification which

Table 1. Audio codecs under evaluation
\begin{tabular}{ccc} 
Codec & bitrate & Frame Size \\
\hline G.711u-a & $64 \mathrm{kbps}$ & $20 \mathrm{~ms}$ \\
GSM & $13 \mathrm{kbps}$ & $20 \mathrm{~ms}$ \\
iLBC & $13.33 \mathrm{kbps}$ & $30 \mathrm{~ms}$ \\
SPEEX & $2.15-24.6 \mathrm{kbos}$ & $40 \mathrm{~ms}$ \\
\hline Sampling frequency at $8 \mathrm{KHz}$ in all cases.
\end{tabular}

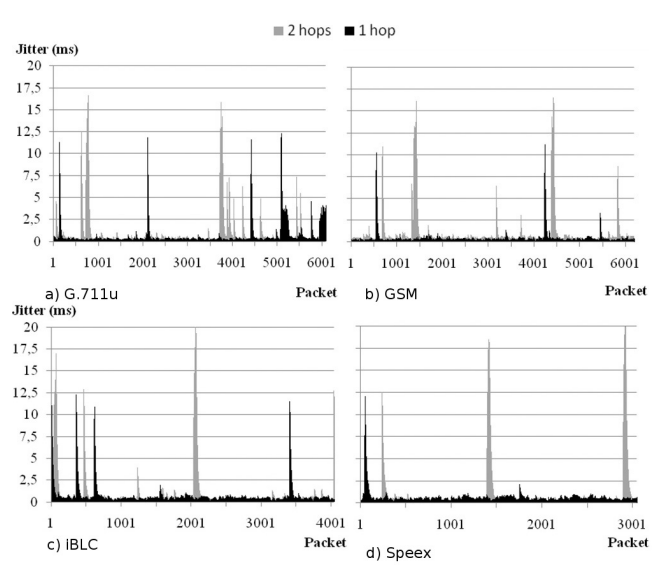

Figure 5. Jitter

reduces the bitrate when necessary. It is a codec oriented toward VoIP, and not toward telephones, for which reason it is optimized for a high quality voice service with low bitrates. Fig. 5 show the jitter for the same audio transmission for 2 minutes for each of the codecs mentioned. As can be seen, the jitter remains constant for one as well as two hops. For G711u, the jitter remains at around $0.25 \mathrm{~ms}$ for one hop and $0.48 \mathrm{~ms}$ for two hops. In GSM, it remains at around $0.28 \mathrm{~ms}$ for one hop and 0.31 for two hops. For iLBC it is 0.41 for one hop, and 0.43 for two hops, and for SPEEX $0.39 \mathrm{~ms}$ for one hop, and 0.42 for two. The variations in this harmonic means are negligible, with maximum peaks of $20 \mathrm{~ms}$ for two hops for iLBC and SPEEX. An important detail to consider is the quantity of packets generated in each codec, for the same time period. With G.711u and GSM we can see 6000 packets received, which corresponds to the 120 seconds transmission time between 20 ms per frame. With iLBC 4000 frames were generated, each containing $30 \mathrm{~ms}$, and with SPX 3000 frames, each containing 40 ms. Table 2 shows the maximum separation between consecutive packets, the average and maximum jitter and the packets loss. From one to two hops, there is an increase in the average and maximum jitter, but this value still remains well below the thresholds for VoIP. The loss of packets does not exceed $0.06 \%$ in any of the cases, which is also well below the limit of $1 \%$.

Table 2. Audio results summary

\begin{tabular}{ccccc} 
1 hop & G.711u-a & GSM & iLBC & SPEEX \\
\hline Max $\delta$ & 47.98 & 139.79 & 149 & 148 \\
Max Jitter & 4.24 & 12.33 & 12.3 & 12.07 \\
Mean Jitter & 0.3 & 0.6 & 0.66 & 0.62 \\
Packets Lost & $0.03 \%$ & $0.02 \%$ & $0.05 \%$ & $0.06 \%$ \\
2 hops & G.711u-a & GSM & iLBC & SPEEX \\
\hline Max $\delta$ & 123.37 & 150.63 & 152 & 148 \\
Max Jitter & 18.02 & 16.66 & 20.26 & 20.63 \\
Mean Jitter & 1.3 & 0.79 & 0.86 & 0.82 \\
Packets Lost & $0.03 \%$ & $0.03 \%$ & $0.00 \%$ & $0.03 \%$ \\
\hline \multicolumn{4}{c}{ all datas in ms, except lost packets. }
\end{tabular}




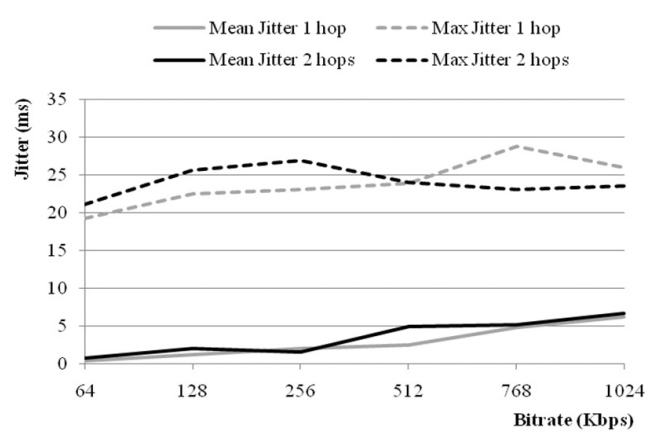

Figure 6. Jitter/bitrare relationship in video streaming

\subsection{Video Traffic}

Recommended quality parameters are 4 seconds of latency, a jitter depending on the buffering of the system and a loss rate of 5\% [7]. Minimizing the need for buffering in the system improves the streaming delay, making the transmission and visualization of the video take place in practically real time. Asterisk supports codecs H261, H.263, H.263+ and H.264. This last one is included in the latest version of Asterisk, but does not have as much compatibility with clients software, so H.263+ was chosen. This codec works with most video-conference applications, offers high image quality with a high level of compression, and has significant improvements in efficiency and robustness over its predecessors. The tests were carried out using different codification rates and the same 2 minutes video sequence. Fig. 6 shows the test results. Even for transmissions $1 \mathrm{Mbps}$, the jitter remains at around $6.7 \mathrm{~ms}$, and the bursts remain below $30 \mathrm{~ms}$, which is extremely low. Carrying out more exhaustive tests using transmission rates of $1 \mathrm{Mbps}$, we obtained the instant jitter that can be seen in Fig. 7 for one and two hops. With one hop, the jitter stays around $3.35 \mathrm{~ms}$, with a typical deviation of $2.83 \mathrm{~ms}$. With two hops, the harmonic average is $3.61 \mathrm{~ms}$ with a typical deviation of $2.94 \mathrm{~ms}$. The loss rate in both cases is $0 \%$.

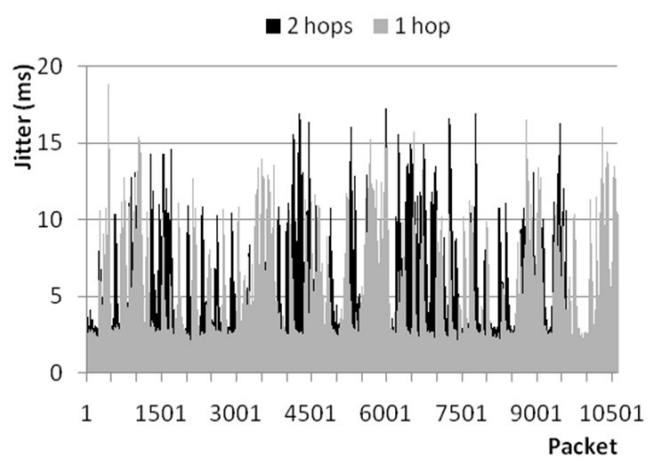

Figure 7. Jitter

\section{Conclusions and Future Work}

The evaluation of control, voice and video traffic in an $802.11 \mathrm{n}$ trunk of one and two hops shows optimum values in all required quality parameters for these types of applications. The differences arising from increasing the trunk from one to two hops are minimal in terms of average jitter and losses, affecting mainly the latency and the throughput. The use of different codecs allows better adaptation to the capacities of the links, using lower bitrates for links with lower throughput, but at the cost of lower quality, although the tests carried out with video-streaming showed a constant flow, without errors, at rates of $1 \mathrm{Mbps}$, offering a high image quality, without cuts or failures in codification, all of which results in a high quality user experience. The evaluation of these parameters in the laboratory allows greater control of the testbed, although in all probability the efficiency of a real installation would be slightly reduced due to external factors, such as an increase in distances, obstacles, interference, climatic factors and industrial noise. Once the viability of this type of traffic over $802.11 \mathrm{n}$ links has been thoroughly checked, it will then be necessary to carry out tests in a real environment, in order to obtain comprehensive conclusions on the performance of this solution for low cost interconnection in urban facilities.

\section{Acknowledgement}

This work was supported by CICYT grant TSI200766637-C02-01/02 and PET2007_0316, which are partially funded by FEDER

\section{References}

[1] A. Gómez-Extremera. Implementar modulo de QoS para VoIP en SIP. Final Thesis Report, Sept. 2006.

[2] IEEE 802.11 Working Group. 802.11n-2009 IEEE Standard for Information technology. Amendment 5: Enhancements for Higher Throughput.

[3] M. Karam and F. Tobagi. Analysis of the delay and jitter of voice traffic over the internet. Proceedings of Infocom, 2001.

[4] J. Rosenberg, H. Schulzrinne, J. A. Camarillo, J. Peterson, R. H. Sparks, and E. Schooler. SIP: Session Initiation Protocol. IETF RFC 3261, Jun. 2002.

[5] H. Schulzrinne, S. Casner, R. Frederick, and V. Jacobson. RTP A Transport Protocol for Real-Time Applications. IETF RFC 1889, Jan. 1996.

[6] J. Silvestre, V. Sempere, and T. Albero. Wireless Metropolitan Area Networks for Telemonitoring Applications. 7th IFAC Int. Conf. on Fieldbuses, Networks in Industrial and Embedded Systems, Nov. 2007.

[7] C. Szigeti, T. and Hattingh. End-to-End QoS Network Design: Quality of service in LANs, WANs and VPNs. Cisco Press, Nov. 2004. 